to their respective Health Ministers and other officials. This Canada-wide network is tied to the World Health Organization and to a global early warning system for emerging pathogens.

Although today these networks function on a sometimes ad hoc basis, the Pan-Canadian Public Health Network (www.phn-rsp.ca) serves to formalize and strengthen our public health infrastructure, which in turn can support you, the health care practitioner. This network facilitates the sharing of information and best practices, fosters the development of collaborative public health strategy, and provides advice, coordinated support and aid to health officials and jurisdic- tions in need. Indeed, the streamlined and unified approach exemplified by the Pan-Canadian Public Health Network will be especially critical during emergencies such as an influenza pandemic. In the absence of a specific crisis, the network will concentrate on other important public health issues, such as health promotion, and chronic disease and injury prevention.

The next big challenge, which is beginning to be addressed through the Pan-Canadian Public Health Network, is to bring together community-based clinicians, public health authorities, viral laboratories and all levels of government to safeguard the health of Canadians against emerging infectious diseases. Clinical suspicion, early recognition and diagnosis, and reporting suspicious cases to the public health system is a vital and concrete way of protecting not only the health of the individual, but also the health of the community at large.

\section{David Butler-Jones}

Public Health Agency of Canada

David Butler-Jones, a former clinician, local Medical Officer of Health in Ontario and Chief Medical Health Officer for Saskatchewan, is Canada's first Chief Public Health Officer and heads the Public Health Agency of Canada.

This article has been peer reviewed.

Competing interests: None declared.

\section{Clinical Vistas BRiefs}

\section{What's your call?}

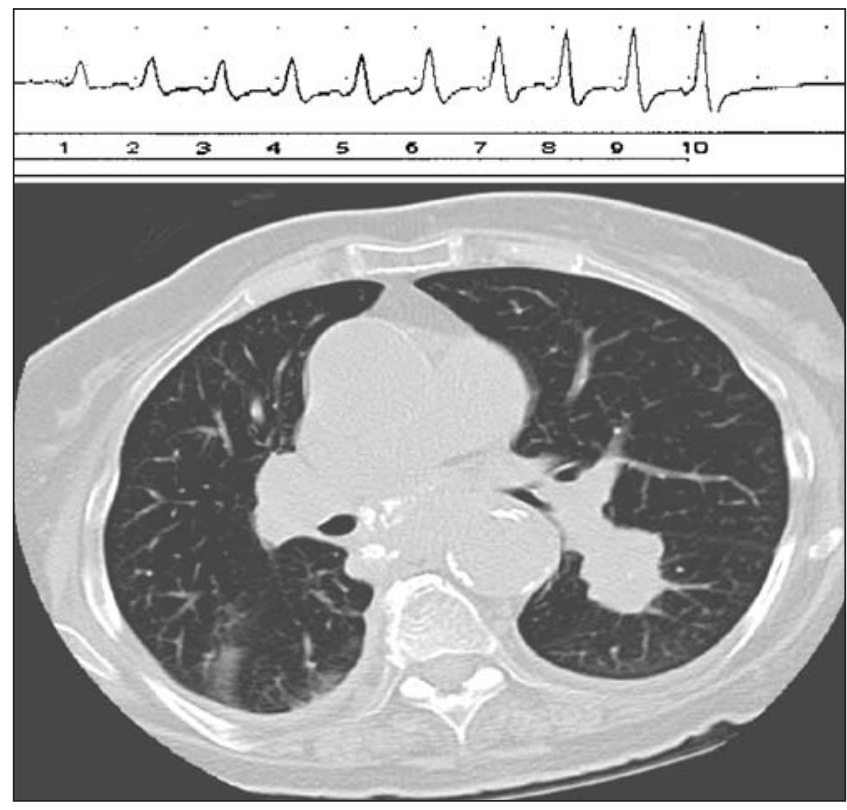

A 72-year-old female smoker presented with morning fatigue, discrete left ptosis and absent reflexes in lower limbs. Top panel: Electromyogram showing evoked motor response of abductor of the fifth finger after stimulation of the left ulnar nerve with a $50-\mathrm{mA}$ current at $50 \mathrm{~Hz}$. Bottom panel: $\mathrm{CT}$ of thorax of the same patient 5 years later.

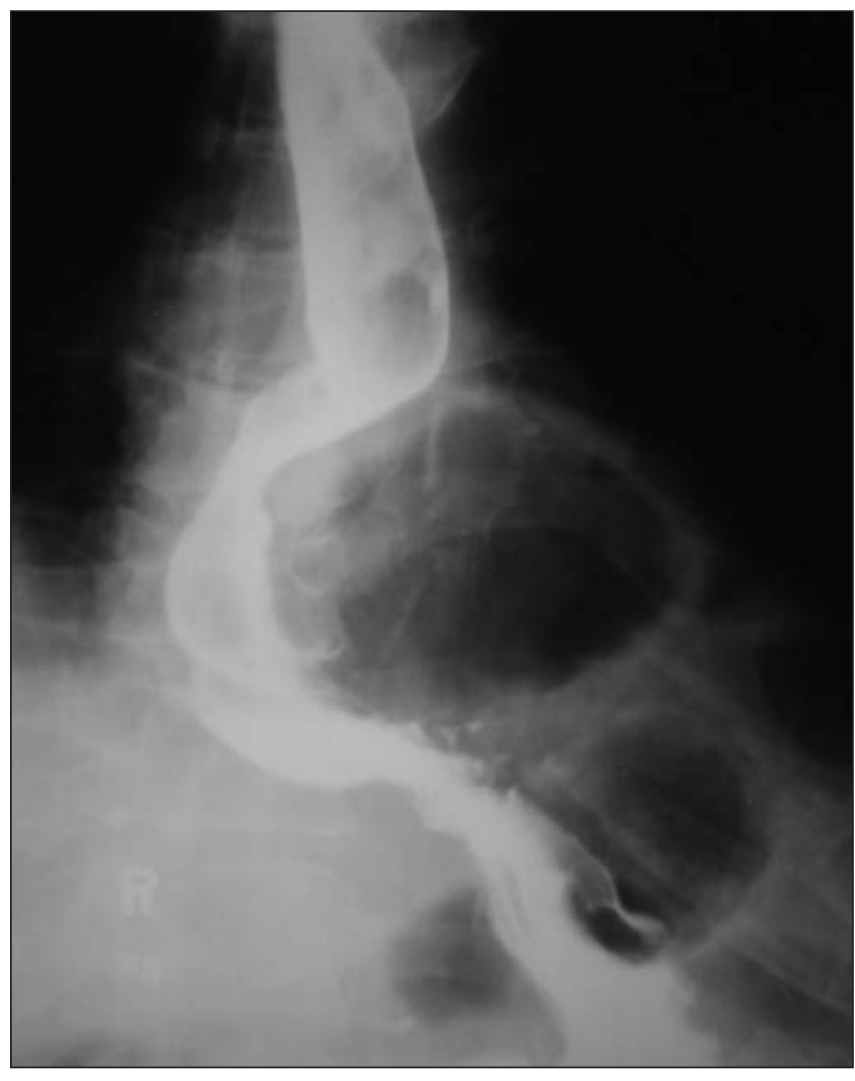

Radiograph with barium swallow from a 6o-year-old emaciated man who presented with nonprogressive dysphagia of 8 months' duration. 


\section{Lambert-Eaton}

\section{myasthenic syndrome}

Repetitive electric stimulation at $50-\mathrm{Hz}$ applied to the ulnar nerve at the wrist normally elicits from the abductor of the fifth finger an evoked response of $\geq 5 \mathrm{mV}$, which may increase no more than $50 \%$. In the case we describe, the electromyogram (EMG) showed that the initial lowamplitude potential was at $0.54 \mathrm{mV}$ and increased progressively to $\mathrm{I} .82 \mathrm{mV}$, a change of $237 \%$. This EMG pattern is a diagnostic feature of Lambert-Eaton myasthenic syndrome (LEMS).

When the patient first presented, she had an unremarkable plain chest radiograph. An initial CT of the thorax showed noncalcified mediastinal lymphadenopathy. Lymph node biopsy revealed a malignant neuroendocrine tumour, but the patient refused treatment. Five years later, a repeat CT of the thorax (Fig. I) showed calcified subcarinal lymphadenopathy (small arrow) and a new 3.I-cm nodule in the left lower lobe (large arrow). These findings are consistent with a primary bronchogenic carcinoma, which had not been apparent initially. The level of antibodies to the P/Q-type voltage-gated calcium channels was high on presentation, at 1595 (normal < 20) pmol/L; it was still elevated 5 years later, at 88I pmol/L.

LEMS is most often a paraneoplastic syndrome associated with small-cell carcinoma, which it may precede. Like myasthenia gravis, it is an autoimmune disorder. Unlike myasthenia gravis, the weakness improves with exertion, and oculobulbar involvement is rare except for ptosis.

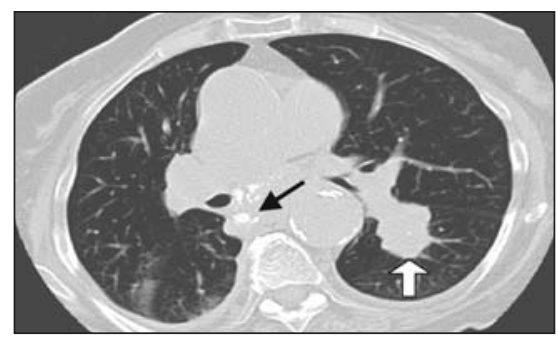

Fig. 1: CT of thorax 5 years after presentation, showing calcified subcarinal lymphadenopathy (black arrow) and new 3.1$\mathrm{cm}$ nodule in left lower lobe (white arrow).
As in our case, EMG is diagnostic of LEMS when it shows incremental increases in muscle action potential in response to repetitive nerve stimulation. EMG helps to differentiate LEMS from other causes of cancer-associated weakness, such as myopathy and polyneuropathy. Detection of antibodies to the P/Qtype voltage-gated calcium channels expressed by the tumour and present on the presynaptic neuron of the neuromuscular junction is also diagnostic of LEMS.

\section{Monique D'Amour}

Department of Neurology

Gilles Gariepy

Department of Pathology

Joseph Braidy

Department of Respiratory Diseases

Hôpital Saint-Luc

Centre hospitalier universitaire

de Montréal

Montréal, Que.

Competing interests: None declared.

DOI:I0.1503/cmaj.060767

\section{Paraesophageal hernia}

Paraesophageal hernia occurs when a portion of the gastric fundus herniates through the diaphragm along the distal esophagus. Why such hernias form is unclear, but they are likely congenital in origin or secondary to a weakness or defect in the phrenoesophageal membrane, especially following surgery in the splenic bed. Paraesophageal hernias differ from sliding hiatus hernias in that they are less common and do not involve a displacement of the gastroesophageal junction.

In general, paraesophageal hernias are asymptomatic. However, patients may present with epigastric or substernal pain or fullness, nausea and dysphagia. Complications such as incarceration, gastric volvulus and perforation are rare but are the reasons why elective surgery is sometimes performed prophylactically.

In cases of paraesophageal hernia, radiography with barium swallow will show a portion of the gastric fundus herniating along the distal esophagus (Fig. I). In our case, a delay in entry of barium into the herniated sac was not discernible

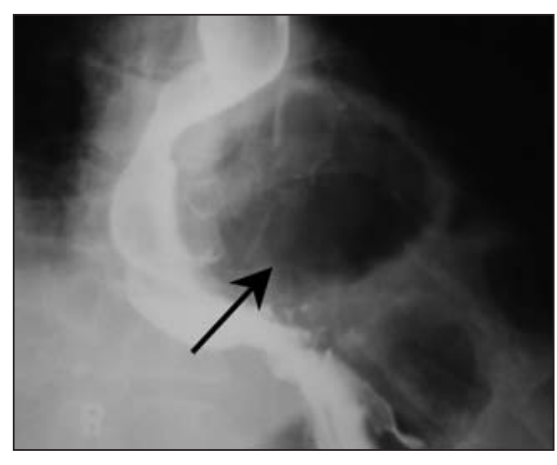

Fig. 1: Herniation of the gastric fundus (arrow) into the thoracic cavity and compression of the distal esophagus.

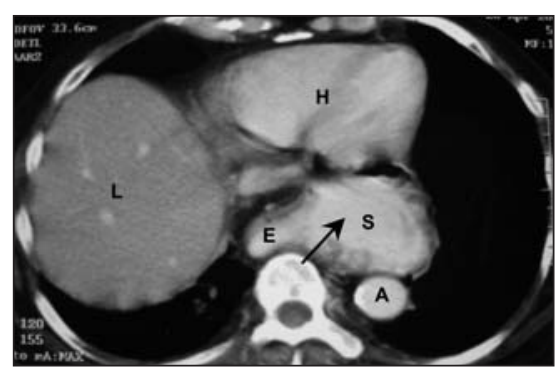

Fig. 2: CT scan of thorax showing herniation of stomach into the thoracic cavity (arrow). $\mathrm{L}=$ liver, $\mathrm{H}=$ heart, $\mathrm{E}=$ esophagus, $\mathrm{S}=$ stomach, $\mathrm{A}=$ aorta.

during fluoroscopy. Demonstration of the gastric cardia below the diaphragm differentiates paraesophageal hernias from sliding hiatus hernias. On CT scans enhanced with contrast material, paraesophageal hernias can be distinguished from other thoracic lesions (e.g., lower esophageal duplications cysts, lower thoracic aortic aneurysms and neuroenteric cysts) by their appearance as a mediastinal mass filled with barium and air located behind the heart and along the esophagus (Fig. 2). In some cases patients may have both a sliding hiatus hernia and a paraesophageal hernia, with radiographic features of both.

\section{Joye Varghese}

Venkataraman Jayanthi

Department of Medical Gastroenterology Stanley Medical College Hospital Chennai, India

Competing interests: None declared.

DOI:Io.I503/cmaj.060952 